\title{
A Mathematical Model for the Leukocyte Filtration Process
}

\author{
Anton Bruil, Tom Beugeling, and Jan Feijen* \\ University of Twente, Department of Chemical Technology, P.O. Box 217, \\ 7500 AE Enschede, The Netherlands
}

Received March 1, 1994/Accepted September 13, 1994

Leukocyte filters are applied clinically to remove leukocytes from blood. In order to optimize leukocyte filters, a mathematical model to describe the leukocyte filtration process was developed by modification of a general theoretical model for depth filtration. The model presented here can be used to predict the time-dependent leukocyte filtration as a function of cell-cell interaction in the filter, filter efficiency, filter capacity, filter dimensions, and leukocyte concentration in the suspension applied to the filter. The results of different leukocyte filtration experiments previously reported in the literature could be well described by the present model. (c) 1995 John Wiley \& Sons, Inc.

Key words: depth filtration - mathematical model • leukocyte filtration - filter efficiency

\section{INTRODUCTION}

Leukocyte filtration has become a popular method to prepare leukocyte poor blood components for clinical use, and nowadays a variety of specialized filters is commercially available for this purpose ${ }^{39}$ Thus far, the optimization of leukocyte filters has been achieved mainly by trial and error, and the mechanisms involved in the filtration process have only recently been investigated in more detail. The development of a mathematical model to describe the filtration process, based on current knowledge of filtration mechanisms, may be helpful to explain results obtained with leukocyte filtration and to optimize leukocyte filters.

Filtration processes can be subdivided in three categories: surface filtration, cake filtration, and depth filtration. ${ }^{5,6}$ The filtration of leukocytes from blood by means of a leukocyte filter can be regarded as a depth filtration process, because the retention of leukocytes by the filter is not restricted to the filter surface but may occur at any place inside the filter bed. Depth filters are most frequently used in industry, in particular to purify wastewater or smoke gases. Studies reported to describe depth filtration processes by mathematical models have almost been exclusively used to describe applications in the field of chemical engineering and include macroscopic descriptions based on a phenomenological approach, ${ }^{1,22,24,37}$ microscopical descriptions based on a theoretical approach, ${ }^{11,26,29,36}$ as well as stochastic models ${ }^{9,15,25,30}$ and network theories. ${ }^{21,32,33,40}$ Most of these

\footnotetext{
* To whom all correspondence should be addressed.
}

studies are, however, very specific and their significance with respect to leukocyte filtration is therefore probably poor.

Only a few attempts to mathematically describe the leukocyte filtration process are known. Diepenhorst ${ }^{7}$ has evaluated a mathematical model, originally derived to explain the removal of ferrous hydroxide particles from groundwater through sand beds, to describe the filtration of leukocytes through cotton wool filters, but he did not succeed in explaining his experimental results. Recently, Prins and Steneker ${ }^{28}$ have developed a computer model to explain the depletion of leukocytes in filters composed of filter segments with different leukocyte trapping efficiencies. With the use of this model, they were able to simulate a number of different filtration experiments. A shortcoming of the model, however, was that the model parameters were based on empirically derived probability factors, and therefore the theoretical value of the model is only small. Moreover, the statistical basis of the model reduces its adaptivity in process engineering and product design.

The aim of the study presented here was to develop a mathematical model to describe the leukocyte filtration process. For this purpose we have adapted a phenomenological depth filtration model from the literature and used it to explain the results of previously reported leukocyte filtration experiments. The results of this study may be important for both the design of new filter materials and the optimization of the filtration process.

\section{THEORY}

A basic hypothesis of depth filtration of the particles to be filtered is that the particle concentration decrease per unit of filter length $(\partial c / \partial h)$ is proportional to the concentration

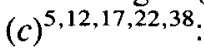

$$
-\frac{\partial c}{\partial h}=\lambda c
$$

The filtration coefficient $\lambda$ is a measure for the efficiency of the filter and was first introduced by Iwasaki ${ }^{19}$ in 1937 . The value of the coefficient depends on the properties of the carrier fluid, the suspension of particles, and the porous filter medium. Various authors have proposed expressions for $\lambda$ as a function of filtration parameters, such as pore 
size, particle size, fluid viscosity, and fluid flow rate. ${ }^{10,14,35}$ The fundamental interest and the applicability of such expressions in leukocyte filtration are small, however, since they were derived semi-empirically with regard to specific process conditions.

During filtration, $\lambda$ changes as a function of time, because the amount of material already deposited in the filter, $\sigma$, influences the filtration of other particles. A general assumption mentioned in the literature is that a limited number of retention sites $\left(\sigma_{\max }\right)$ in the filter is accessible for the capture of particles. Therefore, the filter efficiency decreases upon occupation of these retention sites during filtration. Hence, a linear decrease of $\lambda$ with $\sigma$ has been assumed by several authors ${ }^{12,13,24}$ :

$$
\lambda=\lambda_{0}\left(1-\frac{\sigma}{\sigma_{\max }}\right)
$$

in which $\lambda_{0}$ is the filtration coefficient of the noncontaminated filter. Thus, the value of $\lambda_{0}$ depends purely on the properties of the filter material.

On the other hand, the filter efficiency may increase upon clogging of the filter. During leukocyte filtration, deposited platelets may enhance the retention of leukocytes from the blood cell suspension. ${ }^{4}$ Moreover, material deposited in the filter may reduce the apparent pore size in the filter, thus improving the filter efficiency. ${ }^{2}$ Some authors have proposed a linear expression for $\lambda$ as a function of $\sigma$, in which a factor $\xi$ accounts for the positive effect of particle deposition in the filter on the filter efficiency ${ }^{16,19}$.

$$
\lambda=\lambda_{0}\left(1+\xi \frac{\sigma}{\sigma_{\max }}\right)
$$

Several other relations between $\lambda$ and $\sigma$ have been reported in the literature. ${ }^{14,17,18,35}$ We postulate that for leukocyte filters $\lambda$ can be formulated by a linear combination of Eqs. (2) and (3):

$$
\lambda=\lambda_{0}\left(1-\frac{\sigma}{\sigma_{\max }}\right)\left(1+\xi \frac{\sigma}{\sigma_{\max }}\right)
$$

Thus, the effects of filter properties (first term; $\lambda_{0}$ ), filter saturation (second term), and cell-cell interaction in the filter (third term) are included. In this respect, the constant factor $\xi$ can be defined as a cell-cell interaction parameter, which accounts for the positive effects of deposited cells on the retention of other cells. The interaction between leukocytes and adherent platelets in the filter ${ }^{4}$ can be incorporated in $\xi$ by assuming a constant ratio between the amount of deposited leukocytes and platelets.

An expression for the leukocyte concentration in the filtrate as a function of time $\left(c / c_{0}\right.$ vs. $\left.t\right)$ can be derived from the mass balance for particles in a filter element with thickness $\partial h$ :

$$
\frac{\partial \sigma}{\partial t}+w \frac{\partial c}{\partial h}=0
$$

Here, the fluid flow rate through the filter, $w$, is expressed in unit distance per unit time. The volume flow rate can eventually be calculated by multiplication of $w$ with the planar filter surface area $A$. When $\xi=0$, Eq. (4) simplifies to Eq. (2), and an analytical solution of the differential system [Eqs. (1), (2), and (5)] can be obtained by application of the solution method described by Herzig et al. ${ }^{14}$ :

$$
\frac{c}{c_{0}}=\frac{1}{1+\left[\exp \left(\lambda_{0} h\right)-1\right] \exp \left(-\lambda_{0} w c_{0} t / \sigma_{\max }\right)}
$$

A similar expression for $c / c_{0}$ was also reported by Heertjes and Zuideveld, ${ }^{13}$ who successfully used the equation to describe the filtration of monosized polystyrene particles through a depth filter consisting of a bed of glass spheres.

Another simplification can be made when $\xi=1$. In this case, an analytical solution of the differential system can again be obtained ${ }^{14}$ :

$$
\begin{aligned}
& \frac{c}{c_{0}}= \\
& \frac{\frac{\left[1+\operatorname{ex}^{\prime}\left(2 \lambda_{0} w c_{0} t / \sigma_{\max }\right)\right]}{\left[\exp \left(2 \lambda_{0} w c_{0} t / \sigma_{\max }\right)-1\right]}}{\sqrt{1+\left(\left\{\frac{\left[1+\exp \left(2 \lambda_{0} w c_{0} t / \sigma_{\max }\right)\right]}{\left[\exp \left(2 \lambda_{0} w c_{0} t / \sigma_{\max }\right)-1\right]}-1\right) \exp \left(2 \lambda_{0} h\right)\right.}}
\end{aligned}
$$

In the general case, in which the value of $\xi$ is variable, the differential system cannot be solved analytically. However, we propose a numerical solution from the implicit function of $\mathrm{\sigma}$ :

$$
\sigma=\frac{\sigma_{h=0} \exp \left(-\lambda_{0} h\right)}{\left[\frac{\left(1-\sigma_{h=0} / \sigma_{\max }\right)}{\left(1-\sigma / \sigma_{\max }\right)}\right]^{1 /(1+\xi)}\left[\frac{\left.1+\xi \sigma_{h=0} / \sigma_{\max }\right)}{\left(1+\xi \sigma / \sigma_{\max }\right)}\right]^{\xi /(1+\xi)}}
$$

with

$$
\sigma_{h=0}=\sigma_{\max } \frac{\exp \left[(1+\xi) \lambda_{0} w c_{0} t / \sigma_{\max }\right]-1}{\exp \left[(1+\xi) \lambda_{0} w c_{0} t / \sigma_{\max }\right]+\xi}
$$

Because $c / c_{0}=\sigma / \sigma_{\max },{ }^{14}$ the value of $c / c_{0}$ can be numerically determined from Eqs. (8) and (9).

\section{RESULTS AND DISCUSSION}

The theory developed to describe the leukocyte filtration process can be used to simulate the effect of some filtration parameters on the filter efficiency and to explain the results of filtration experiments. It should be kept in mind, however, that the expressions derived for $\sigma$ and $c / c_{0}$ as a function of $t$ [Eqs. (6 to 9)] were derived by assuming a constant flow rate $w$ during the filtration, which is not always confirmed in practice. During filtration, clogging may lead to a progressive pressure drop across the filter resulting in a slow decrease of the flow rate as a function of time. The 
theory of pressure drop of the suspension fluid through the filter has been well developed in the literature, ${ }^{14,18,35}$ but this aspect has not been included in the current study. During routine leukocyte filtration in blood banks, overloading of the filter and consequent filter clogging do not usually occur, and the flow generally remains constant. In this case it is possible to calculate the filtered volume $\left(V_{t}\right)$ of the blood cell suspension through a filter from the filter surface area $A$ and the superficial filtration rate $w$ :

$$
V_{t}=A w t
$$

This approach allows us to draw plots of $c / c_{0}$ against the filtered volume $V_{t}$, which is common use in the field of leukocyte filtration research.

\section{Simulation Experiments}

A model simulation will be performed in which cell-cell interaction in the filter occurs. Deposited cells or cell aggregates may reduce the apparent pore size in the filter, thus improving the filter efficiency. ${ }^{2}$ Moreover, adherent platelets in the filter may enhance the retention of granulocytes from the blood cell suspension. ${ }^{4}$ Also indirect effects, such as the activation of the complement system, which may lead to leukocyte adhesion, ${ }^{20}$ probably influences the filtration process. To account for all of these effects together, one single cell-cell interaction parameter $\xi$ was introduced. When the contribution of cell-cell interaction to the filtration mechanism becomes more important, the value of $\xi$ should be increased. When, however, cell-cell interaction is not considered to be important in leukocyte filtration, $\xi$ should be taken as zero. The relation of $\xi$ with specific cell-cell interaction phenomena occurring in the filter requires further research, and until then the quantification of $\xi$ should be estimated by trial and error.

Using the present theory, the effect of cell deposition in the filter on the efficiency of the leukocyte filtration process was predicted (Fig. 1). Two extreme cases, determined by the filtration coefficient of the noncontaminated filter, should be distinguished. When the leukocyte removal efficiency of the noncontaminated filter is relatively large, i.e., when $c / c_{0}$ is close to zero in the beginning of the filtration process, the effect of cell-cell interaction on the filter efficiency becomes important only after prolonged continuation of the filtration (Fig. 1A). In this case, the effect of cell-cell interaction is demonstrated by a shift of the breakthrough point, i.e., the filtered volume after which an increase of the leukocyte content in the filtrate can be measured, to a larger volume of the filtrate. This theoretical finding was confirmed by experimental results reported by Steneker et al. ${ }^{34}$ who showed that the breakthrough point during filtration of leukocytes was achieved earlier in the absence of platelets in the cell suspension than in the presence of platelets.

A distinct effect of cell-cell interaction was predicted for the hypothetical case that the leukocyte removal efficiency of the noncontaminated filter is relatively small, i.e., when $c / c_{0}$ is substantially larger than zero in the beginning of the
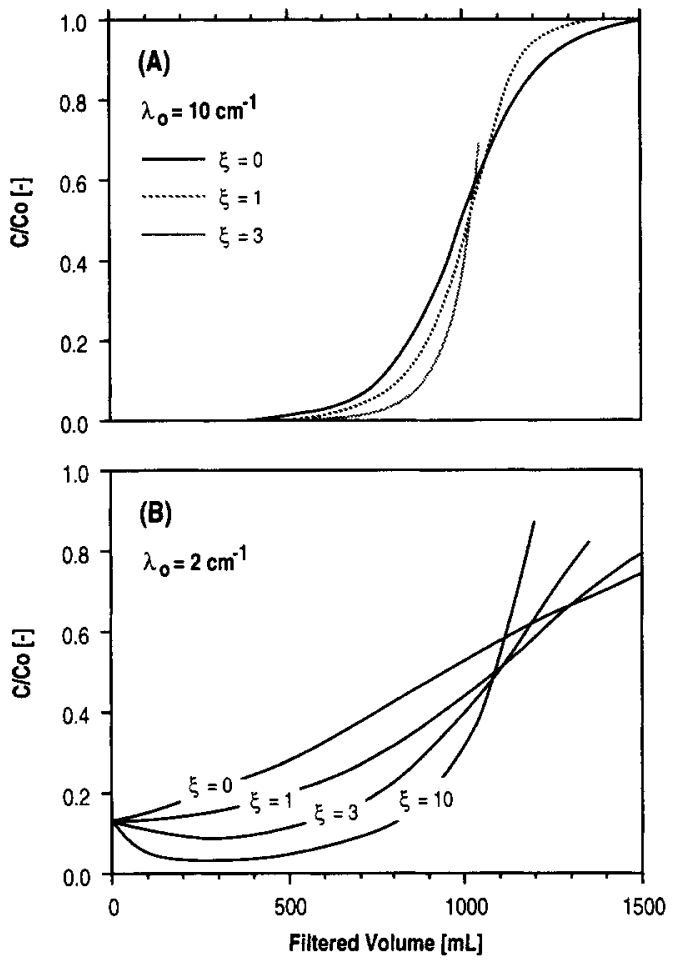

Figure 1. Theoretical effect of deposited cells in the filter on the course of the leukocyte filtration process using a filter with (A) a high initial filtration coefficient $\left(\lambda_{0}=10 \mathrm{~cm}^{-1}\right)$ and (B) a low initial filtration coefficient $\left(\lambda_{0}=2 \mathrm{~cm}^{-1}\right)$. The value of the cell-cell interaction parameter $\xi$ was varied between 0 and 10 . Other parameters were fixed and set at $c_{0}=$ $10^{6}$ leukocytes $\cdot \mathrm{cm}^{-3}, \sigma_{\max }=10^{7}$ leukocytes $\cdot \mathrm{cm}^{-3}, w=10^{-2}$ $\mathrm{cm} \cdot \mathrm{s}^{-1}, h=1 \mathrm{~cm}$, and $A=100 \mathrm{~cm}^{2}$.

filtration (Fig. 1B). In this case, an increased filter efficiency becomes apparent already at the onset of the filtration. This effect was best demonstrated for values of $\xi$ larger than 1, resulting in an initial decrease of the concentration of leukocytes in the filtrate. In spite of a larger filter efficiency at higher $\xi$, saturation of the filter occurs earlier. This is obviously due to the fact that the maximum uptake of leukocytes in the filter is determined by the filter capacity $\sigma_{\max }$, which is independent of $\xi$, as imposed by the present theoretical model. It should be noted, however, that an initial increase of filter efficiency is not likely to occur in leukocyte filtration practice.

Currently available filters, used in standard blood bank procedures, are designed to remove at least $99 \%$ of leukocytes during the initial stage of the filtration, ${ }^{31}$ and, hence, the effects of cell-cell interaction do not become apparent in these filters. Besides, commercially applied filter materials will probably have a high filter efficiency $\lambda_{0}$ compared to the effective filtration efficiency of a dense layer of depleted cells $\left(\lambda_{\text {cells }}\right)$, so a value for $\xi$ larger than 1 cannot be obtained in practice. Nonetheless, in a few cases with model filters, which were not typically designed for high leukocyte removal rates, we have observed a slight increase of the filtration efficiency during the filtration process. ${ }^{2}$

Another application of the present model is to simulate the effects of filtration parameters and process conditions on 
the concentration of leukocytes in the filtrate. Filtration curves were drawn in cases where specific model parameters were varied, whereas other model parameters were kept constant at a value approximating their order of magnitude in filtration practice (Fig. 2). In all cases, it was assumed that the effects of cell-cell interaction in the filter are small compared to the leukocyte retention in the filter material itself, and thus $\xi \approx 0$. It was found that an optimal value of the initial filtration coefficient $\lambda_{0}$ is a prerequisite for efficacious removal of leukocytes from the cell suspension (Fig. 2A). This finding confirms that filter properties, such as the pore size, porosity, roughness, charge, wettability, and physicochemical properties of the filter surface, are of prime importance for optimal filter functioning.

Many authors have derived semiempirical relations for $\lambda_{0}$ as a function of the filter properties, ${ }^{10,14,35}$ but this was not a particular subject of the study presented here. However, an important prediction is mentioned here. Since $\lambda_{0}$ can be regarded as a leukocyte trapping probability in the noncontaminated filter ${ }^{14}$ and the mechanism of leukocyte filtration is probably governed by cell adhesion to the filter material, ${ }^{2,3}$ it is reasonable to postulate that $\lambda_{0}$ is proportional to the specific surface area $(S)$ of the leukocyte filter. A similar relation between $\lambda_{0}$ and $S$ was also proposed for other depth filtration processes by other authors. ${ }^{17,23}$ Assuming that $S$ is inversely proportional to the filter pore size $d,{ }^{2}$ we obtain

$$
\lambda_{0} \sim \frac{1}{d}
$$

In contrast to $\lambda_{0}$, the filter capacity $\sigma_{\max }$ (Fig. 2B) and the leukocyte concentration in the suspension applied to the filter, $c_{0}$ (Fig. 2C), do not affect the initial extent of leukocyte removal. These parameters primarily determine the filtered volume at the breakthrough point. Note that this point, as well as the initial extent of leukocyte removal, is easily adjusted by the filter thickness $h$ (Fig. 2D). This finding is important with respect to filter design, since it implies that in principle each given filter material, irrespective of its $\lambda_{0}$ and $\sigma_{\max }$ coefficients, can be applied to construct a leukocyte filter. The nominal filter thickness $h^{*}$ required to prepare a leukocyte-poor suspension with a permitted leukocyte concentration $c^{*}$ can be derived by taking $t=0$ in Eq. (6), resulting in

$$
h^{*}=\frac{1}{\lambda_{0}} \ln \left(\frac{c_{0}}{c^{*}}\right)
$$

In addition to this nominal filter thickness, a layer with thickness $\Delta h^{*}$ should be added to shift the breakthrough point to the desired volume $V^{*}$ of the leukocyte-poor filtrate. For $\exp \left(\lambda_{0} h^{*}\right) \gg 1$, it can be derived from Eqs. (6) and (10) that $\Delta h^{*}$ is proportional to $V^{*}$, according to

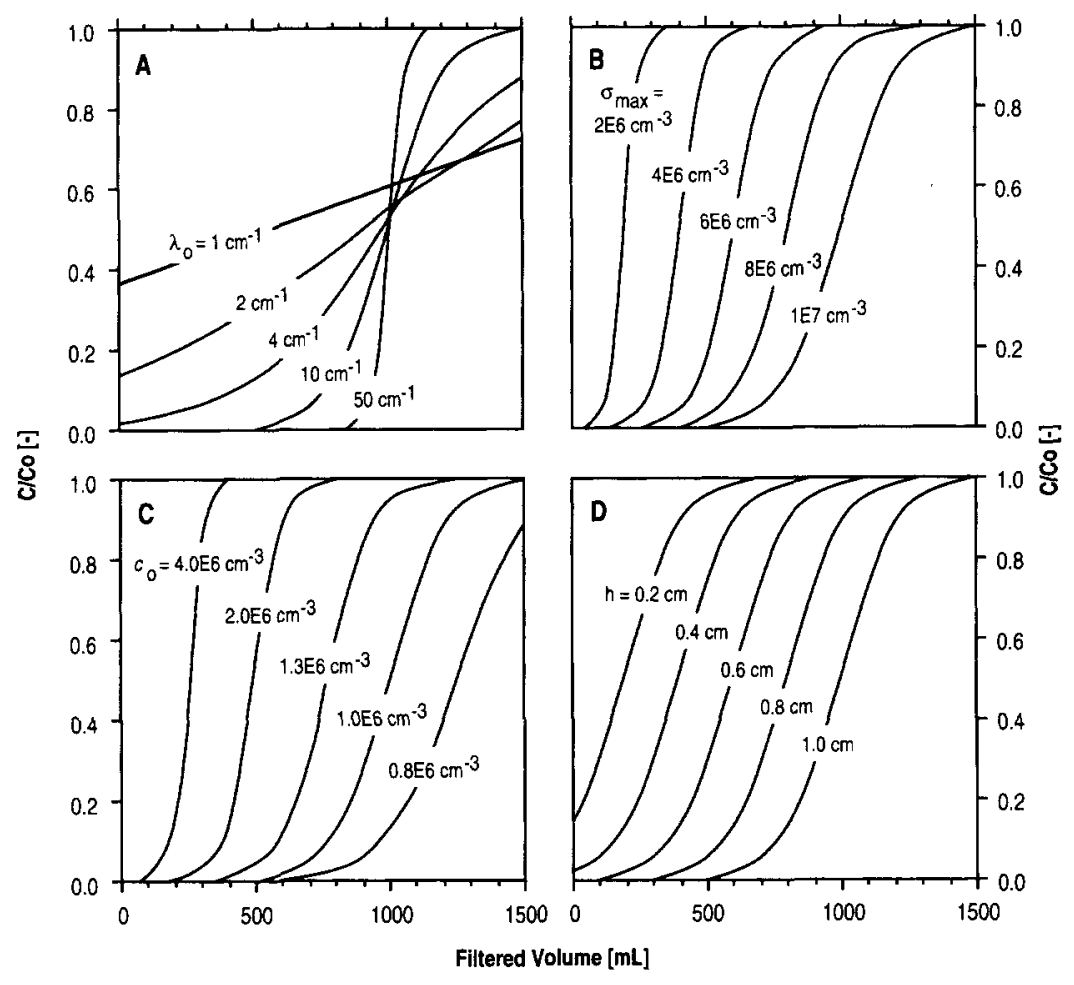

Figure 2. Theoretical effect of some filtration parameters on the course of the leukocyte filtration process. Plots in the graph show the effect of (A) initial filtration coefficient $\lambda_{0}$, (B) filter capacity $\sigma_{\max },(\mathrm{C})$ leukocyte concentration of the cell suspension applied to the filter $c_{0}$, and (D) filter thickness $h$. The effect of cell-cell interaction in the filter was neglected $(\xi=$ 0 ). Fixed parameters were set at $c_{0}=10^{6}$ leukocytes $\cdot \mathrm{cm}^{-3}, \lambda_{0}=10 \mathrm{~cm}^{-1}, \sigma_{\max }=10^{7}$ leukocytes $\cdot \mathrm{cm}^{-3}, w=10^{-2} \mathrm{~cm} \cdot \mathrm{s}^{-1}, h=1 \mathrm{~cm}$, and $A=100 \mathrm{~cm}^{2}$. 


$$
\Delta h^{*}=\frac{c_{0}}{A \sigma_{\max }} V^{*}
$$

Thus, when the filter parameters $\lambda_{0}$ and $\sigma_{\max }$ as well as the leukocyte concentration $c_{0}$ in the suspension applied to the filter are known, the dimensions of the filter, $A$ and $h$, can be optimized by using Eqs. (12) and (13). The value of $\lambda_{0}$ and $\sigma_{\max }$ can be determined by curve fitting of filtration characteristics.

\section{Curve Fitting Experiments}

To describe the results of filtration experiments by means of the general mathematical formulation for the filtration process [Eqs. (8) and (9)], at least three filtration parameters, $\xi, \sigma_{\max }$ and $\lambda_{0}$, should be solved independently. Since theoretical relations for these parameters in terms of the properties of the filter are not yet well developed, this can only be achieved by trial and error. The problem is simplified, however, when the effects of cell-cell interaction in the filter are neglected compared to the leukocyte retention by the filter material itself. In this case, when $\xi \approx 0$, the filtration process is described by Eq. (6), and the parameters $\sigma_{\max }$ and $\lambda_{0}$ can be determined from the results of filtration experiments.

The filter parameter $\sigma_{\max }$ can be calculated from the estimated half-value volume $\left(V_{1 / 2}\right)$ at which $c / c_{0}=0.5$, according to a method described by Heertjes and Zuideveld. ${ }^{13}$ For $\exp \left(\lambda_{0} h\right) » 1$, it can be derived from Eqs. (6) and (10) that

$$
\sigma_{\max }=\frac{c_{0}}{A h} V_{1 / 2}
$$

When $\sigma_{\max }$ is known, $\lambda_{0}$ can be calculated from the linear relationship between $\ln \left[\left(c_{0} / c\right)-1\right]$ and $V_{t}$, derived from Eqs. (6) and (10):

$$
\ln \left(\frac{c_{0}}{c}-1\right)=-\frac{c_{0 \lambda 0}}{A \sigma_{\max }} V_{t}+\ln \left[\exp \left(\lambda_{0} h\right)-1\right]
$$

Equations (14) and (15) were used to fit the results of filtration experiments reported in the literature with the present filtration model. It should be noted that the present model was specifically developed for symmetric filters, in which $\lambda_{0}$ is constant. Eventually, asymmetric filters can be described by regarding them as a series of symmetric filter layers. For the sake of comprehension, however, the examples given here deal with symmetric filter types only.

Leukocyte filtration data reported for a cotton wool filter designed by Diepenhorst et al. ${ }^{8}$ could be well described by the present theory (Fig. 3). Since values of filtration parameters $c_{0}, h, A$, and $w$ were accurately given by the authors, the value of $\sigma_{\max }$ and $\lambda_{0}$ could be precisely determined by using Eqs. (14) and (15). Nevertheless, a distinct deviation from the model occurred at large filtration volumes. The discrepancy between theory and data is probably

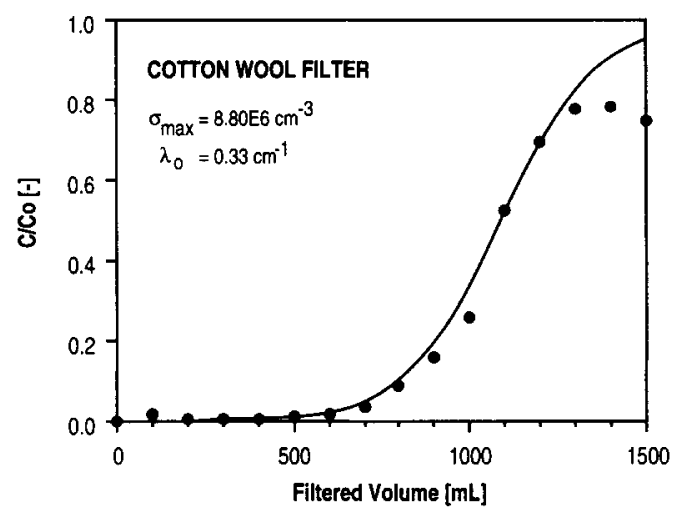

Figure 3. Theoretical fit of a leukocyte filtration curve obtained by filtration of a blood cell suspension through an experimental cotton wool filter. Experimental data from ref. 8. Fixed parameters were set at $c_{0}=5$ $\times 10^{6}$ leukocytes $\cdot \mathrm{cm}^{-3}, \xi=0, w=3 \times 10^{-2} \mathrm{~cm} \cdot \mathrm{s}^{-1}, h=25 \mathrm{~cm}$, and $A=25 \mathrm{~cm}^{2}$.

due to the effect of leukocyte-containing microaggregates, which are formed spontaneously during storage of leukocyte-containing blood. ${ }^{27}$ Microaggregates (40 to $100 \mu \mathrm{m}$ ) are significantly larger than leukocytes $(5$ to $15 \mu \mathrm{m})$, and, therefore, two different filtration mechanisms may occur simultaneously. Leukocyte depletion is mainly caused by adhesion, whereas microaggregates will be captured by sieving. ${ }^{2}$ Because large leukocyte-containing microaggregates are easily retained by the filter, especially in the latter stage of the filtration when the filter becomes clogged, the leukocyte recovery $\left(c / c_{0}\right)$ cannot become complete. Nowadays it is common in blood bank practice to prevent microaggregate formation by partial removal of the leukocytes from blood prior to storage. ${ }^{27}$

Remarkably, in contrast with our results, attempts of Diepenhorst et al. ${ }^{7}$ to explain their results with a depth filtration model adapted from Heertjes and Lenk ${ }^{12}[\mathrm{Eq}$. (16)] were unsuccessful:

$$
\frac{c}{c_{0}}=\frac{1}{1+\left[\exp \left(-w c_{0} k t\right)\right]\left[\exp \left(k \epsilon_{0} h\right)-1\right]}
$$

The authors do not provide the backgrounds of their model nor do they give an explanation for its failure. We assume, however, that a major shortcoming of their model is the need of a value for the initial filter porosity $\epsilon_{0}$ whereas our model requires a value for the initial filter capacity $\sigma_{\max }$, which is determined experimentally. By taking $\epsilon_{0}$ as an approximation for $\sigma_{\max }$, Diepenhorst et al. ${ }^{7}$ assumed that the complete free filter volume is available for leukocyte depletion, which is not very likely. Since the Diepenhorst et al. model is based on an equation with only one degree of freedom (i.e., $k \equiv \lambda_{0} / \epsilon_{0}$ ), whereas the present model requires two degrees of freedom $\left(\lambda_{0}\right.$ and $\left.\sigma_{\max }\right)$, the latter model allows a better fit of experimental data.

Filtration data for two commercial filters were reported by Reesink et al., ${ }^{31}$ but a specification of the filter dimensions $A$ and $h$ as well as the filtration rate $w$ was not given 
by these authors. Their results were successfully explained by the present theory, assuming values of $A, h$, and $w$ equal to specifications described for the above-mentioned cotton wool filtration experiment (Fig. 4). Using these values, it was concluded from the fit parameters $\sigma_{\max }$ and $\lambda_{0}$ that the capacity of the Imugard filter (Fig. 4A: $\sigma_{\max } \approx 3.00 \times 10^{6}$ leukocytes $\cdot \mathrm{cm}^{-3}$ ) is lower than the capacity of the Erypur filter (Fig. 4B: $\sigma_{\max } \approx 3.65 \times 10^{6}$ leukocytes $\cdot \mathrm{cm}^{-3}$ ). The leukocyte filtration efficiency $\lambda_{0}$ was found to be practically the same for both filters. A similar conclusion, albeit not supported by quantitative filter indices, was also stated by Reesink et al. ${ }^{31}$ in their original study.

The theory applied to the leukocyte filter process mentioned above can principally not be used to explain the results of previously reported leukocyte filtration experiments with a series of polyurethane membranes differing in pore size. ${ }^{2}$ These experiments were performed at variable flow rate $w$, due to substantial clogging of the filters during filtration, whereas the present theory was derived for constant values of $w$. In most cases, however, the value of $c / c_{0}$ did not significantly change during the course of the filtration, ${ }^{2}$ and change of the time axis to account for a decreasing flow rate will not significantly affect the filtration profile. It is, therefore, assumed that the deviation of experimental data from the predictions based on the present theoretical model will not be very large. Indeed, some of the membrane filtration characteristics could be success-
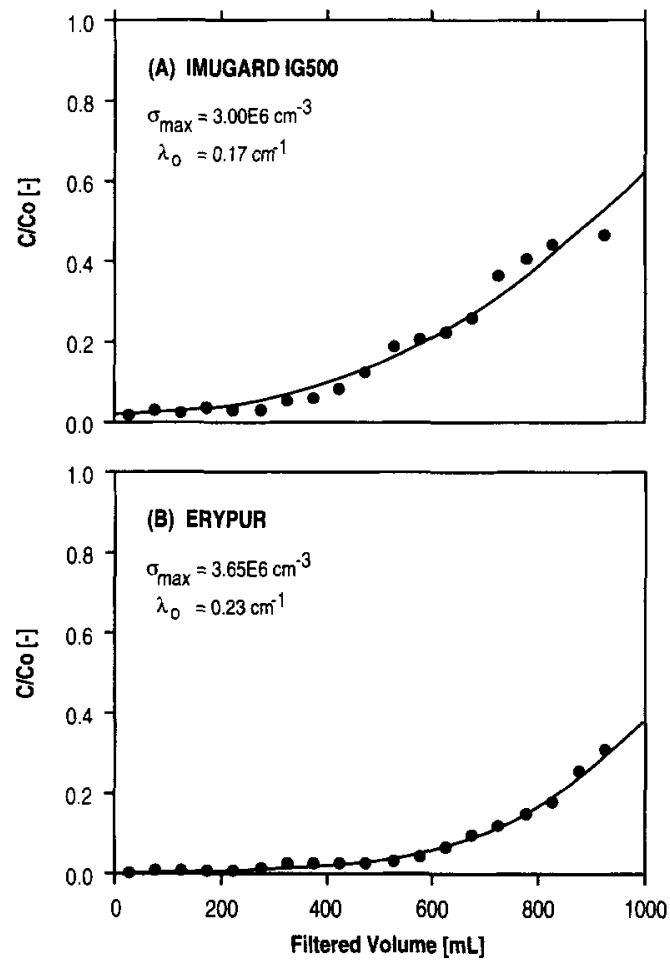

Figure 4. Theoretical fit of leukocyte filtration curves obtained by filtration of blood cell suspensions through commercially available leukocyte filters: (A) Imugard IG500 and (B) Erypur filter. Experimental data from ref. 31. Fixed parameters were set at $c_{0}=2.1 \times 10^{6}$ leukocytes $\cdot \mathrm{cm}^{-3}$, $\xi=0, w=0.03 \mathrm{~cm} \cdot \mathrm{s}^{-1}, h=25 \mathrm{~cm}$, and $A=25 \mathrm{~cm}^{2}$.

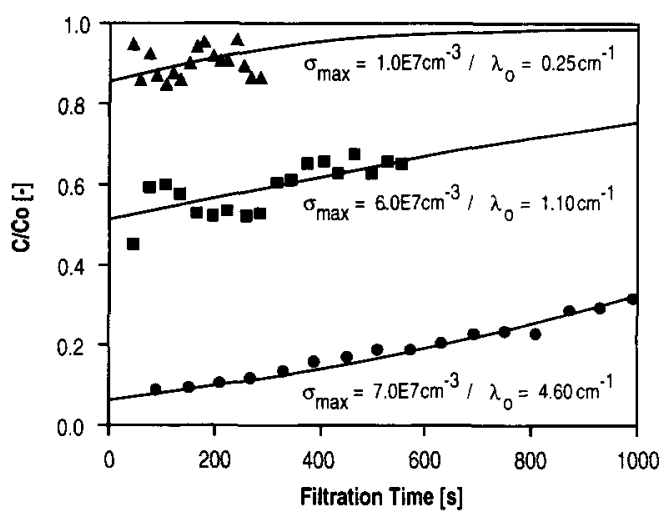

Figure 5. Theoretical fit of leukocyte filtration curves obtained by filtration of blood cell suspensions through membrane filters with different average pore sizes: $19 \mu \mathrm{m}$ (circles), $30 \mu \mathrm{m}$ (squares), or $43 \mu \mathrm{m}$ (triangles). Data from previously reported experiments. ${ }^{2}$ Fixed parameters were set at $c_{0}=6 \times 10^{6}$ leukocytes $/ \mathrm{mL}, \xi=0, h=0.6 \mathrm{~cm}, A=25 \mathrm{~cm}^{2}$, and $w$ $=5 \times 10^{-3}(19-\mu \mathrm{m}$ filter $), 10^{-2}(30-\mu \mathrm{m}$ filter $)$, or $2 \times 10^{-2}(43-\mu \mathrm{m}$ filter) $\mathrm{cm} \cdot \mathrm{s}^{-1}$.

fully described by Eq. (6) (Fig. 5). Values of the filtration coefficient $\lambda_{0}$, found for membrane filters with different pore sizes, agree well with the relation $\lambda_{0} \sim d^{-1}$ [Eq. (11)]. Hence, a good comparison of different leukocyte filter materials can be accomplished by application of the present model.

\section{CONCLUSIONS}

The objective of this study was to derive a mathematical model to explain the leukocyte filtration process. It was found that most of the phenomena observed during leukocyte filtration experiments could be explained by a general mathematical formulation of the process [Eqs. (8) and (9)]. Simplification of this theory, by neglecting the explicit effects of cell-cell interaction in the filter on the filter efficiency, allowed a quantitative description of the filtration process. This theory [Eq. (6)] can be used to quantitatively compare the filtration characteristics of different filter materials and to calculate the dimensions of a leukocyte filter composed of a specific material. Moreover, the present filtration model can be used to explain and optimize the function of current leukocyte filters.

\section{NOMENCLATURE}

A filter surface area $\left(\mathrm{cm}^{2}\right)$

c concentration of leukocytes in cell suspensions $\left(\mathrm{cm}^{-3}\right)$

$c_{0}$ concentration of leukocytes in cell suspension before filtration $\left(\mathrm{cm}^{-3}\right)$

$c^{*} \quad$ permitted concentration of leukocytes in filtrate $\left(\mathrm{cm}^{-3}\right)$

$d$ pore size $(\mathrm{cm})$

$h$ filter thickness $(\mathrm{cm})$

$h^{*} \quad$ nominal filter thickness to prepare filtrate with leukocyte concentration $c^{*}(\mathrm{~cm})$

$k \quad$ filtration constant in Diepenhorst model, Eq. (16) $\left(\mathrm{cm}^{-1}\right)$

$S \quad$ specific filter surface area $\left(\mathrm{cm}^{-1}\right)$

$t$ time (s)

$V_{t} \quad$ filtered volume of cell suspension as function of time $\left(\mathrm{cm}^{3}\right)$ 
$V^{*} \quad$ desired volume of leukocyte poor filtrate $\left(\mathrm{cm}^{3}\right)$

$w \quad$ superficial filtration velocity $\left(\mathrm{cm} \cdot \mathrm{s}^{-1}\right)$

$\epsilon_{0} \quad$ filter porosity in Diepenhorst model, Eq. (16) (dimensionless)

$\lambda$ filtration coefficient $\left(\mathrm{cm}^{-1}\right)$

$\lambda_{0}$ filtration coefficient of noncontaminated filter $\left(\mathrm{cm}^{-1}\right)$

$\sigma \quad$ concentration of leukocytes in filter $\left(\mathrm{cm}^{-3}\right)$

$\sigma_{\max }$ filter capacity, i.e., maximum leukocyte uptake by filter $\left(\mathrm{cm}^{-3}\right)$

$\xi \quad$ cell-cell interaction parameter (dimensionless)

\section{References}

1. Adin, A., Rebhun, M., 1987. Deep-bed filtration: Accumulationdetachment model parameters. Chem. Eng. Sci. 42: 1213-1219.

2. Bruil, A., van Aken, W. G., Beugeling, T., Feijen, J., Steneker, I., Huisman, J. G., Prins, H. K. 1991. Asymmetric membrane filters for the removal of leukocytes from blood. J. Biomed. Mater. Res. 25: 1459-1480.

3. Bruil, A., Oosterom, H. A., Steneker, I., Al, B. J. M., Beugeling, T., van Aken, W. G., Feijen, J. 1993. Poly(ethyleneimine) modified filters for the removal of leukocytes from blood. J. Biomed. Mater. Res. 27: 1253-1268.

4. Bruil, A., Sheppard, J. I., Feijen, J., Feuerstein, I. A. 1994. In vitro leukocyte adhesion to modified polyurethane surfaces. III. Effect of flow, fluid medium, and platelets on PMN adhesion. J. Biomater. Sci. Polymer Edn. 5: 263-277.

5. Cain, C. W. 1988. Filtration theory. pp. 4.3-4.8 In: P. A. Schweitzer (ed.), Handbook of separation techniques for chemical engineering. McGraw-Hill, New York.

6. Dickey, G. D. 1961. Theory of filtration. pp. 24-33 In: Filtration. Rheinhold, New York.

7. Diepenhorst, P. 1974. Removal of leukocytes from whole blood and erythrocyte suspensions by filtration through cotton wool. VII. Studies on possible mechanisms. pp. 86-90 In: P. Diepenhorst, Removal of Leukocytes from Blood by Filtration through Cotton Wool. Ph.D. Dissertation, Delft University of Technology, Delft, The Netherlands.

8. Diepenhorst, P., Sprokholt, R., Prins, H. K. 1972. Removal of leukocytes from whole blood and erythrocyte suspensions by filtration through cotton wool. I. Filtration technique. Vox. Sang. 23: 308-320.

9. Fan, L. T., Hwang, S. H., Nassar, R., Chou, S. T. 1985. An experimental study of deep-bed filtration: Stochastic analysis. Powder Technol. 44: 1-11.

10. Gosh, M. M. 1975. Physicochemical approach to water and waste water filtration. J. Environ. Eng. Div. 101: 71-86.

11. Guzy, C. J., Bonano, E. J., Davis, E. J. 1983. The analysis of flow and colloidal particle retention in fibrous porous media. J. Colloid Interface Sci. 95: 523-543.

12. Heertjes, P. M., Lerk, C. F., 1967. The functioning of deep-bed filters. Part II: The filtration of flocculated suspensions. Trans. Inst. Chem. Engr. 45: T138-T145.

13. Heertjes, P. M., Zuideveld, P. L. 1978. Clarification of liquids using filter aids. Part II. Depth filtration. Powder Technol. 19: 31-43.

14. Herzig, J. P., LeClerc, D. M., Le Goff, P. 1970. Flow of suspensions through porous media-Application to deep filtration. Ind. Eng. Chem. 62: 8-35.

15. Hsu, E. H., Fan, L. R. 1984. Experimental study of deep bed filtration: A stochastic treatment. AlChE J. 30: 267-273.

16. Ives, K. J. 1970. Depth filtration of liquids, Filtr. Separ. 7: 700-704.

17. Ives, K. J. 1975. Mathematical models of deep bed filtration. pp. 203-224 In: K. J. Ives (ed.), The scientific basis of filtration. Noordhoff International, Leiden.

18. Ives, K. J. 1985. Deep bed filters. NATO ASI Ser. 88: 90-149.
19. Iwasaki, T. 1937. Some notes on sand filtration. J. Am. Water Works Assoc. 29: 1591-1602.

20. Kazatchkine, M. D., Carreno, M. P. 1988. Activation of the complement system at the interface between blood and artificial surfaces. Biomaterials 9: 30-35.

21. Leitzelement, M., Maj, P., Dodds, J. A., Greffe, L. J. 1984. Deep bed filtration in a network of random tubes. pp. 273-296 In: J. Gregory (ed.), Solid-liquid separation. Wiley, New York.

22. Mackie, R. I. 1989. Rapid gravity filtration-Towards a deeper understanding. Filtr. Separ. 26: 32-35.

23. Mackrle, V., Mackrle, S. 1961. Adhesion in filters. J. Sanit. Eng. Div. Proc. Am. Soc. Civil. Eng. SA5: 17-32.

24. Matharasan, R., Apelian, D., Romanowski, C. 1981. Laboratory investigation of aluminum filtration through deep-bed and ceramic open-pore filters. J. Metals 33: 12-18.

25. Nassar, R., Chou, S. T., Fan, L. T. 1986. Modelling and simulation of deep-bed filtration: Stochastic compartment model. Chem. Eng. Sci. 41: 2017-2027.

26. Netter, P., Conti, C. 1986. Efficiency of industrial filters for molten metal treatment. Evaluation of a filtration process model. Light Metals 2: $847-860$.

27. Prins, H. K., de Bruijn, J. C. G. H., Hinrichs, H. P. J., Loos, J. A. 1980. Prevention of microaggregate formation by removal of "buffy coats." Vox Sang. 39; 48-51.

28. Prins, H. K., Steneker, I. 1992. Statistic simulation of leukocyte depletion by filtration with the aid of a computer model. pp. 97-109 In: I. Steneker, Leukocyte depletion from fresh red cell concentrates by fiber filtration. Ph.D. Dissertation, Free University of Amsterdam, Amsterdam, The Netherlands.

29. Rajagopalan, R., Tien, C. 1976. Trajectory analysis of deep-bed filtration with the sphere-in-cell porous media model. AlChE J. 22: $523-533$.

30. Ramarao, B. V., Tien, C. 1988. Stochastic simulation of aerosol deposition in model filters. AlChE J. 34: 253-262.

31. Reesink, H. W., Veldman, H., Henrichs, H. J., Prins, H. K., Loos, J. A. 1982. Removal of leukocytes from blood by fibre filtration. A comparison study on the performance of two commercially available filters. Vox Sang. 42: 281-288.

32. Rege, S. D., Fogler, H. S. 1988. A network model for deep bed filtration of solid particles and emulsion drops. AlChE J. 34: 1761-1772.

33. Sharma, M. M., Yortsos, Y. C. 1987. A network model for deep bed filtration processes. AlChE J. 33: 1644-1653.

34. Steneker, I., Prins, H. K., Florie, M., Loos, J. A., Biewenga, J. 1992. Mechanisms of white cell reduction in red cell concentrates by filtration. The effect of the cellular composition of the red cell concentrates. Transfusion 32: 42-50.

35. Tien, C., Payatakes, A. C. 1979. Advances in deep bed filtration AlChE J. 25: 737-759.

36. Vaidyanathan, R., Tien, C. 1988. Hydrosol deposition in granular beds. Chem. Eng. Sci. 43: 289-302.

37. Vigneswaran, S., Tulachan, R. K. 1988. Mathematical modelling of transient behaviour of deep bed filtration. Wat. Res. 22: 1093-1100.

38. Vinten, A. J. A., Nye, P. H. 1985. Transport and deposition of dilute colloidal suspensions in soils. J. Soil Sci. 36: 531-541.

39. Wenz, B. 1990. Clinical and laboratory precautions that reduce the adverse reactions, alloimmunization, infectivity, and possibly immunomodulation associated with homologous transfusions. Transfusion Med. Rev. 4(suppl. 1): 3-7.

40. Yortsos, Y. C., Sharma, M. 1986. Application of percolation theory to noncatalytic gas-solid reactions. AlChE J. 32: 46-55. 